\title{
On Robust Stability for Hurwitz Polynomials via Recurrence Relations and Linear Combinations of Orthogonal Polynomials
}

\author{
Alejandro Arceo $\left(\mathbb{D},{ }^{1}\right.$ Héctor F. Flores $\left(\mathbb{D},{ }^{2}\right.$ Lino G. Garza $\left(\mathbb{D},{ }^{3}\right.$ Luis E. Garza $(\mathbb{D}),{ }^{2}$ \\ and Gerardo Romero iD 1 \\ ${ }^{1}$ Universidad Autónoma de Tamaulipas, UAM Reynosa Rodhe, Reynosa, Tamaulipas, Mexico \\ ${ }^{2}$ Universidad de Colima, Colima, Mexico \\ ${ }^{3}$ Universidad de Monterrey, San Pedro Garza García, Nuevo León, Mexico
}

Correspondence should be addressed to Lino G. Garza; lino.garza@udem.edu

Received 20 October 2021; Accepted 11 January 2022; Published 2 March 2022

Academic Editor: Alejandro F. Villaverde

Copyright (c) 2022 Alejandro Arceo et al. This is an open access article distributed under the Creative Commons Attribution License, which permits unrestricted use, distribution, and reproduction in any medium, provided the original work is properly cited.

In this contribution, we use the connection between stable polynomials and orthogonal polynomials on the real line to construct sequences of Hurwitz polynomials that are robustly stable in terms of several uncertain parameters. These sequences are constructed by using properties of orthogonal polynomials, such as the well-known three-term recurrence relation, as well as by considering linear combinations of two orthogonal polynomials with consecutive degree. Some examples are presented.

\section{Introduction and Background}

1.1. Hurwitz Polynomials and Robust Stability. A polynomial with real coefficients is called a Hurwitz polynomial if and only if all of its roots have negative real part. The study of Hurwitz polynomials is motivated by the role they play in the analysis of the stability of linear systems of the form

$$
\dot{y}(t)=\mathbf{A} y(t), \quad y \in \mathbb{R}^{n}, \mathbf{A} \in \mathbb{R}^{n \times n},
$$

where $\mathbf{A}$ is a fixed matrix that represents some process and $\dot{y}(t)$ denotes the derivative of the vector $y(t)$ componentwise. In fact, it is very well-known that Hurwitz polynomials characterize the stability of linear invariant-time continuous systems. More precisely, such a linear system is asymptotically stable (i.e., its solutions tend to zero as $t \longrightarrow \infty$ ) if and only if the characteristic polynomial $\operatorname{det}(\mathbf{A}-x \mathbf{I})$, where $\mathbf{I}$ denotes the identity $n \times n$ matrix, is a Hurwitz polynomial [1-3]. As a consequence, many algebraic properties of Hurwitz (also called stable) polynomials have been studied in the literature, including many characterizations in order to determine whether or not a given real polynomial is Hurwitz without computing its roots explicitly. Among the most widely known criteria for determining the stability of a given polynomial, we can mention the Hurwitz-Routh test [4] and the stability test [5]. Other important characterizations are the Hermite-Biehler theorem [6] and Markov's parameters theorem [2]. The latter two characterizations play a central role in the relation between Hurwitz polynomials and orthogonal polynomials, as it will be discussed in the sequel.

On the other hand, in most of real-life applications, the physical process represented by equation (1) has uncertainty. This uncertainty can be caused by the inherent nature of the process under analysis, by the difficulty of taking precise measurements on the variables of the process, or a combination of both phenomena. As a consequence, it is useful to introduce an uncertain parameter on the mathematical representation of the linear system. In this situation, the system is represented by

$$
\dot{y}(t)=\mathbf{A}(\mathbf{q}) y(t)
$$

where $\mathbf{q}$ is a $d$-dimensional vector with $d$ parameters defined in some domain $D \subset \mathbb{R}^{d}$, and the system is said to be robustly stable if $\operatorname{det}(\mathbf{A}(\mathbf{q})-x \mathbf{I})$ is a degree invariant Hurwitz polynomial for every $\mathbf{q} \in D$. Different types of uncertainty are considered in the literature. For instance, the uncertain structure defined by 


$$
p(x, \mathbf{q})=q_{n} x^{n}+q_{n-1} x^{n-1}+\ldots+q_{0}, \quad q_{i} \in\left[a_{i}, b_{i}\right], a_{i}<b_{i}, i=0, \ldots, n,
$$

where $\mathbf{q}$ is a $(n+1)$-dimensional vector in the rectangular domain $D=\left[a_{0}, b_{0}\right] \times \ldots \times\left[a_{n}, b_{n}\right] \in \mathbb{R}^{n+1}$, is referred to as independent interval uncertainty. Here, the main tool is the celebrated Kharitonov's theorem [7] that establishes that the robust stability of the whole family is equivalent to the stability of just four polynomials, the so-called Kharitonov polynomials. The case where the coefficients of the characteristic polynomial are linear combinations of the parameters $q_{i}$ is called affine linear uncertainty [5] and the fundamental result to determine the robust stability is called Edge's theorem [8]. Polynomic uncertainty occurs when the coefficients of the characteristic polynomial are multivariate polynomials in the parameters $q_{i}$. Finally, nonlinear uncertainty structure is obtained when the coefficients of the polynomial are more general nonlinear functions of the parameters. It is worth mentioning that there are not general theorems to determine the robust stability for polynomic and nonlinear uncertainty, and therefore most of the robustness analysis is carried out by using numerical approaches $[5,9,10]$.

1.2. Orthogonal Polynomials on the Real Line. Given a positive Borel measure $\sigma$ supported on some interval $E \subset \mathbb{R}$, an inner product on the linear space of polynomials can be defined by

$$
\langle p, q\rangle_{\sigma}=\int_{E} p(x) q(x) \mathrm{d} \sigma(x) .
$$

If $E$ is unbounded, we require that the moments $c_{n}=$ $\int_{E} x^{n} \mathrm{~d} \sigma(x)$ are finite for $n \geq 0$. The Gram-Schmidt orthogonalization process guarantees the existence of a sequence of real polynomials $\left\{p_{n}\right\}_{n \geq 0}$ satisfying the orthogonality conditions

$$
\left\langle p_{n}, p_{m}\right\rangle_{\sigma}=\gamma_{n} \delta_{m, n}, \quad \gamma_{n} \neq 0, n, m \geq 0,
$$

where $\delta_{m, n}$ is Kronecker's delta. Such a sequence becomes unique once a suitable normalization is chosen. In this contribution, we will consider a monic sequence denoted by $\left\{P_{n}\right\}_{n \geq 0}$. The second kind polynomials $\left\{Q_{n}\right\}_{n \geq 0}$ are defined by

$$
Q_{n}(x)=\int_{E} \frac{P_{n}(y)-P_{n}(x)}{y-x} \mathrm{~d} \sigma(y),
$$

and play a central role in Padé approximation [11]. It is not difficult to see that $Q_{n}$ is a polynomial of degree $n-1$.

Orthogonal polynomials are a topic of study that dates back to the late 17th century, although a general theory started to flourish in the 18th century with the contributions of Stieltjes [12], Markov [13], and Chebyshev [14], among many others. Nowadays, it constitutes a very active area of research due to their numerous applications in approximation theory [11], numerical integration [15], mathematical physics [16], digital image processing [17, 18], stochastic processes $[19,20]$, signal theory $[21,22]$, and control theory [23], among many others. They have a number of nice properties, including the following [24, 25]:

(1) For any $n \geq 1$, the roots of each $P_{n}$ are real, simple, and located in the interior of the convex hull of $E$.

(2) For any $n \geq 1$, the roots of $P_{n}$ and $P_{n-1}$ interlace. That is, between any two consecutive roots of $P_{n}$, there is exactly one root of $P_{n-1}$.

(3) For any $n \geq 1$, the roots of $P_{n}$ and $Q_{n}$ interlace.

Notice that the first property above means that $P_{n}$ will have real, simple, and positive roots if $E \subset \mathbb{R}_{+}$. On the other hand, $\left\{P_{n}\right\}_{n \geq 0}$ and $\left\{Q_{n}\right\}_{n \geq 0}$ also satisfy the three-term recurrence relations

$$
\begin{gathered}
P_{n+1}(x)=\left(x-b_{n}\right) P_{n}(x)-a_{n} P_{n-1}(x), \quad n \geq 0, \\
Q_{n+1}(x)=\left(x-b_{n}\right) Q_{n}(x)-a_{n} Q_{n-1}(x), n \geq 1,
\end{gathered}
$$

with initial conditions $P_{0}=1, P_{-1}=0, Q_{0}=0, Q(x)=1$, where $\left\{a_{n}\right\}_{n \geq 0}$ and $\left\{b_{n}\right\}_{n \geq 0}$ are a positive and a real sequence of numbers, respectively. Notice that $a_{0}$ is arbitrary and it is usually defined as $a_{0}=c_{0}=\int_{E} \mathrm{~d} \sigma(x)$. A converse result also holds: any sequence $\left\{P_{n}\right\}_{n \geq 0}$ constructed by using equation (7) with arbitrary sequences $\left\{a_{n}\right\}_{n \geq 0}$ (positive numbers) and $\left\{b_{n}\right\}_{n \geq 0}$ (real numbers) will be orthogonal with respect to some positive measure $\sigma$ supported on $\mathbb{R}$. This fact is known as Favard's theorem [24]. Moreover, it is possible to impose conditions on the coefficients $a_{n}$ and $b_{n}$ in order to guarantee that the associated orthogonality measure is supported on $\mathbb{R}$. Namely, this will be the case if and only if $b_{n}>0$ for every $n \geq 0$ and there exists a sequence $\left\{g_{n}\right\}_{n \geq 0}$ satisfying $0<g_{n}<1$ for $n \geq 1,0 \leq g_{0}<1$, and $a_{n} /\left(b_{n} b_{n-1}\right)=g_{n}\left(1-g_{n-1}\right)$ for $n \geq 1$. Any pair of sequences $\left\{a_{n}\right\}_{n \geq 0},\left\{b_{n}\right\}_{n \geq 0}$ satisfying those conditions are said to generate the chain sequence $\left\{a_{n} /\left(b_{n} b_{n-1}\right)\right\}_{n \geq 1}$. As a particular case, this occurs when $\left\{a_{n}\right\}_{n \geq 0}$ and $\left\{b_{n}\right\}_{n \geq 0}$ are chosen in such a way that $a_{n} /\left(b_{n} b_{n-1}\right)=k$ for some fixed $k$ satisfying $0<k \leq 1 / 4$ (see $[24,26])$. Notice that in this case we have $g_{n}=1 / 2$ for every $n \geq 0$.

1.3. Robust Stability via Orthogonality. The relation between Hurwitz polynomials and orthogonal polynomials has been known in the literature for quite some time. They are both closely related with the theory of continued fractions and the moment problem, as pointed out in [2]. Also, in the early nineties, Genin (see [27]) describes a relation between the Euclid's algorithm, the Routh-Hurwitz algorithm, and orthogonal polynomials and establishes that any Hurwitz polynomial can be identified with a (finite) sequence of orthogonal polynomials. This relation has been recently analyzed with more detail in [28] (in the more general framework of matrix orthogonal polynomials) and [26]. Specifically, if $f_{2 n}(x)=x^{2 n}+$ $a_{2 n-1} x^{2 n-1}+\ldots+a_{1} x+a_{0}$ is a (monic) polynomial of even degree $2 n$ and we consider the decomposition 


$$
f_{2 n}(x)=h\left(x^{2}\right)+x g\left(x^{2}\right),
$$

then $h$ is a polynomial of degree $n$ and $g$ is a polynomial of degree at most $n-1$. Indeed, they are the even and odd parts of $f_{2 n}$. Hermite-Biehler theorem states that a polynomial $f(x)=h\left(x^{2}\right)+x g\left(x^{2}\right)$ is Hurwitz if and only if $h$ and $g$ have negative, simple, and interlacing roots, with the rightmost root being that of $h$, and $h(0) g(0)>0$ [4]. In [26] the authors show that, up to a change a variable and multiplication by a constant, $h$ is an orthogonal polynomial with respect to some measure $\sigma$ supported on $\mathbb{R}_{+}$, and $g$ is the associated second kind polynomial. More precisely, we have

$$
P_{n}(x)=(-1)^{n} h(-x), Q_{n}(x)=(-1)^{n-1} g(-x) .
$$

A similar decomposition holds for odd degree Hurwitz polynomials, but now the odd part has the form

$$
\beta P_{n}(x)-Q_{n}(x)=(-1)^{n} g(-x),
$$

where $\beta$ is the constant term that appears in the power expansion of the rational function $g(x) / h(x)$. It is important to mention that the coefficients on this power expansion are precisely the moments of the orthogonality measure $\sigma$. In other words, to every Hurwitz polynomial $f_{2 n}$ (or $\left.f_{2 n+1}\right)$, we can associate a finite sequence of orthogonal polynomials $\left\{P_{k}\right\}_{k=0}^{n}$ and of course their corresponding second kind polynomials $\left\{Q_{k}\right\}_{k=0}^{n}$. More importantly, as a straightforward consequence of the Hermite-Biehler theorem, the following converse result holds.

Theorem 1 (see [26]). Let $\sigma$ be a positive measure supported on $\mathbb{R}_{+}$and denote by $\left\{P_{n}\right\}_{n \geq 0}$ and $\left\{Q_{n}\right\}_{n \geq 0}$ its associated (monic) orthogonal polynomials and second kind polynomials, respectively. Define $G_{n}(x)=Q_{n}(x)+\beta_{n} P_{n}(x)$ with $\beta_{n}>0$ such that the zeros of $P_{n}$ and $G_{n}$ are positive and interlaced. Then,

$$
\begin{aligned}
f_{2 n+1}(x) & =(-1)^{n} x P_{n}\left(-x^{2}\right)+(-1)^{n} G_{n}\left(-x^{2}\right), \quad n \geq 0, \\
f_{2 n}(x) & =(-1)^{n} P_{n}\left(-x^{2}\right)+(-1)^{n-1} x Q_{n}\left(-x^{2}\right), \quad n \geq 1,
\end{aligned}
$$

\section{are Hurwitz polynomials.}

Notice that the construction of the odd degree Hurwitz polynomial $f_{2 n+1}$ in Theorem 1 requires the choice of a parameter $\beta_{n}$ in such a way that the roots of $P_{n}$ and $G_{n}$ interlace. In Proposition 2.2 in [26], it was shown that it is possible to choose $\beta_{n}=\lambda$ (independent of $n$ ) in such a way that the roots of $P_{n}$ and $G_{n}$ interlace for every $n \geq 1$. Indeed, it suffices to take $\lambda>M=\lim _{n \longrightarrow \infty} Q_{n}(0) / P_{n}(0)$, provided such a limit exists. On the other hand, if we fix $m>0$ and define $M=Q_{m}(0) / P_{m}(0)$, then $P_{k}$ and $G_{k}$ will have interlaced roots for all $k=1, \ldots, m$ if $\lambda>M$.

Observe that the previous theorem implies that we can construct a sequence of Hurwitz polynomials by using sequences $\left\{P_{n}\right\}_{n \geq 0}$ and $\left\{Q_{n}\right\}_{n \geq 0}$, when the orthogonality measure is supported on $\mathbb{R}_{+}$; i.e., the zeros of each $P_{n}$ are simple and positive. This property was used in [29] to construct robustly stable sequences of Hurwitz polynomials by using the Laguerre and Jacobi classical orthogonality measures supported on $\mathbb{R}_{+}$ (see [25]). The main idea was to introduce a parameter $t$ on the measure, in such a way that it remains positive. As a consequence, the corresponding inner product, the associated orthogonal sequence, and the second kind polynomials will depend on $t$. That is, $\left\{P_{n}(x, t)\right\}_{n>0}$ will be orthogonal for every value of $t$, and thus the sequence $\left\{f_{n}(x, t)\right\}_{n \geq 1}$ constructed by using Theorem 1 will be Hurwitz for every value of $t$. In other words, if we consider $t$ as an uncertain parameter, the sequence $\left\{f_{n}(x, t)\right\}_{n \geq 1}$ will be robustly stable. It is worth mentioning that robustly stable families constructed in such a way have been already used in the design of a compensator that robustly stabilizes an interval plant with uncertain time-delay (see [30]) and can also be used for applications in control design.

In this contribution, we propose two alternative ways to construct robustly stable sequences of Hurwitz polynomials by using other properties of orthogonal polynomials. The structure of the manuscript is as follows. In Section 2, we introduce an uncertain parameter $t$ on the coefficients of the three-term recurrence relation and then use the Favard theorem described in the previous subsection and Theorem 1 to construct a sequence of Hurwitz polynomials that is robustly stable for every value of $t$ within an specified range. In Section 3, we generate robustly stable sequences by using linear combinations of orthogonal polynomials. Some examples are presented in Section 4. Finally, some conclusions and open problems are discussed in Section 5.

\section{Stable Polynomials Generated by a Recurrence Relation}

In this section, we consider the recurrence relation

$P_{n+1}(x, t)=\left(x-b_{n}(t)\right) P_{n}(x, t)-a_{n}(t) P_{n-1}(x, t), \quad n \geq 0$,

with initial conditions $P_{-1}(x, t)=0$ and $P_{0}(x, t)=1$, and $a_{n}(t), b_{n}(t)$ are functions of an uncertain parameter $t$. If $\left\{a_{n}(t)\right\}_{n \geq 0},\left\{b_{n}(t)\right\}_{n \geq 0}$ generate a chain sequence for all $t \in I$, where $I$ is some interval, then Favard's theorem guarantees that the constructed monic sequence $\left\{P_{n}(x, t)\right\}_{n \geq 0}$ is orthogonal with respect to some positive measure $\sigma$ supported on $\mathbb{R}_{+}$. Thus, the roots of each $P_{n}(x, t)$ will be real, simple, and positive for every value of $t \in I$. Clearly, the associated second kind polynomials $\left\{Q_{n}(x, t)\right\}_{n \geq 0}$ will also be $t$-dependent, and for each $n \geq 2$ the roots of $P_{n}(x, t)$ and $Q_{n}(x, t)$ will satisfy an interlacing property for every value of $t \in I$. As a consequence, we can use Theorem 1 to construct a sequence of polynomials $\left\{f_{n}(x, t)\right\}_{n \geq 1}$ that will be stable for every value of $t \in I$.

Let us start with a simple example. For $n \geq 0$, consider $a_{n}(t)=t$ and

$$
b_{n}(\alpha, t)=\left\{\begin{array}{ll}
t & \text { if } n \text { is odd, } \\
\alpha & \text { if } n \text { is even, }
\end{array} \quad \text { for } n \geq 0\right.
$$

where $t \in I$ with $I=(0, \infty)$ and $\alpha \geq 4$. It is easy to check that $\left\{a_{n}(t)\right\}_{n \geq 0}$ and $\left\{b_{n}(\alpha, t)\right\}_{n \geq 0}$ satisfy 


$$
0<\frac{a_{n}(t)}{b_{n-1}(\alpha, t) b_{n}(\alpha, t)} \leqslant \frac{1}{4}, \quad n \geq 1 .
$$

As pointed out in the previous section, they constitute a chain sequence. Notice that we have introduced two variables $t$ and $\alpha$ that can be considered as uncertain parameters. From our previous discussion, the sequence $\left\{P_{n}(x, \alpha, t)\right\}_{n \geq 0}$ defined by

$$
P_{n+1}(x, \alpha, t)=\left(x-b_{n}(\alpha, t)\right) P_{n}(x, \alpha, t)-a_{n}(t) P_{n-1}(x, \alpha, t), \quad n \geq 0,
$$

with $P_{-1}(x, \alpha, t)=0, P_{0}(x, \alpha, t)=1$, is an orthogonal sequence for all $t>0$ and all $\alpha \geq 4$. Moreover, we can obtain the following closed expressions for these polynomials.
Proposition 1. Let $\left\{a_{n}(t)\right\}_{n \geq 0}$ and $\left\{b_{n}(t)\right\}_{n \geq 0}$ be defined as before. For $n \geq 1, t>0$, and $\alpha \geq 4$, we have

$$
\begin{aligned}
P_{2 n}(x, \alpha, t) & =\sum_{j=0}^{n}(-1)^{j}\left(\begin{array}{c}
2 n-j \\
2(n-j)
\end{array}\right) t^{j}(x-t)^{n-j}(x-\alpha)^{n-j}, \\
P_{2 n-1}(x, \alpha, t) & =\sum_{j=0}^{n-1}(-1)^{j}\left(\begin{array}{c}
2 n-1-j \\
2(n-j)-1
\end{array}\right) t^{j}(x-t)^{n-j}(x-\alpha)^{n-1-j} .
\end{aligned}
$$

Proof. We proceed by induction on $n$. Since we clearly have $P_{1}(x, \alpha, t)=(x-t)$ and $P_{2}(x, \alpha, t)=(x-t)(x-\alpha)-t$, assuming equations (17) and (18) hold for $n \geq 1$, we will prove both of them for $n+1$. Taking into account the properties of the binomial coefficients and equation (16), with $y_{t}=x-t$ and $y_{\alpha}=x-\alpha$, we obtain

$$
\begin{aligned}
P_{2 n+1}(x, \alpha, t)= & y_{t} P_{2 n}(x, \alpha, t)-t P_{2 n-1}(x, \alpha, t), \\
= & y_{t}^{n+1} y_{\alpha}^{n}+\sum_{j=1}^{n}(-1)^{j}\left(\begin{array}{c}
2 n-j \\
2(n-j)
\end{array}\right) t^{j} y_{t}^{n-j+1} y_{\alpha}^{n-j} \\
& +\sum_{j=1}^{n}(-1)^{j}\left(\begin{array}{c}
2 n-j \\
2(n-j)+1
\end{array}\right) t^{j} y_{t}^{n-j+1} y_{\alpha}^{n-j}, \\
= & \left(\begin{array}{c}
2 n+1 \\
2 n+1
\end{array}\right) y_{t}^{n+1} y_{\alpha}^{n}+\sum_{j=1}^{n}(-1)^{j}\left(\begin{array}{c}
2 n+1-j \\
2(n-j)+1
\end{array}\right) t^{j} y_{t}^{n-j+1} y_{\alpha}^{n-j}, \\
= & \sum_{j=0}^{n}(-1)^{j}\left(\begin{array}{c}
2(n+1)-1-j \\
2(n+1-j)-1
\end{array}\right) t^{j} y_{t}^{n+1-j} y_{\alpha}^{n-j},
\end{aligned}
$$

and equation (17) is proved. In a similar way, we get

$$
\begin{aligned}
P_{2 n+2}(x, \alpha, t)= & y_{\alpha} P_{2 n+1}(x, \alpha, t)-t P_{2 n}(x, \alpha, t), \\
= & y_{t}^{n+1} y_{\alpha}^{n+1}+\sum_{j=1}^{n}(-1)^{j}\left(\begin{array}{c}
2 n+1-j \\
2(n+1-j)-1
\end{array}\right) t^{j} y_{t}^{n+1-j} y_{\alpha}^{n+1-j} \\
& +\sum_{j=1}^{n+1}(-1)^{j}\left(\begin{array}{c}
2 n+1-j \\
2(n+1-j)
\end{array}\right) t^{j} y_{t}^{n+1-j} y_{\alpha}^{n+1-j}, \\
= & y_{t}^{n+1} y_{\alpha}^{n+1}+\sum_{j=1}^{n}(-1)^{j}\left(\begin{array}{c}
2 n+2-j \\
2(n+1-j)
\end{array}\right) t^{j} y_{t}^{n+1-j} y_{\alpha}^{n+1-j} \\
& +(-1)^{n+1} t^{n+1},
\end{aligned}
$$


which yields equation (18) when $n$ is replaced by $n+1$.

Of course, we can obtain similar expressions for the second kind polynomial $Q_{n}(x, \alpha, t)$, since they satisfy the same recurrence relation, with different initial conditions.
We state the following result without proof, since it is very similar to the proof of the previous proposition.

Proposition 2. For $n \geq 1, t>0$, and $\alpha \geq 4$,

$$
\begin{aligned}
Q_{2 n+1}(x, \alpha, t) & =\sum_{j=0}^{n}(-1)^{j}\left(\begin{array}{c}
2 n-j \\
2(n-j)
\end{array}\right) t^{j}(x-\alpha)^{n-j}(x-t)^{n-j}, \\
Q_{2 n}(x, \alpha, t) & =\sum_{j=0}^{n-1}(-1)^{j}\left(\begin{array}{c}
2 n-1-j \\
2(n-j)-1
\end{array}\right) t^{j}(x-\alpha)^{n-j}(x-t)^{n-1-j},
\end{aligned}
$$

with $Q_{1}(x, \alpha, t)=1$.

Several observations are in order. First, notice that if we consider $t$ and $\alpha$ as uncertain parameters, the structure of the coefficients in $P_{n}(x, \alpha, t)$ and $Q_{n}(x, \alpha, t)$ indicates that the Hurwitz polynomial constructed via Theorem 1 will have polynomic uncertainty. Second, observe that we can introduce some generality in the previous example. In fact, we can replace $t$ by a more general continuous function $g(t)$ such that $g(t)>0$ for every $t \in I$. For instance, we can consider the case when $a_{n}(t)=g^{2}(t)$ and $b_{n}(\alpha, t)=\alpha g(t)$ with $\alpha \geq 2$. It is easily seen that $\left\{a_{n}(t)\right\}_{n \geq 0}$ and $\left\{b_{n}(\alpha, t)\right\}_{n \geq 0}$ satisfy equation (15) and, as a consequence, they generate a chain sequence. Thus, again by using equation (16), we can construct a sequence of orthogonal polynomials that depend on $t$ and $\alpha$. As before, we can get explicit expressions for their coefficients.

Proposition 3. For $n \geq 0$, let $a_{n}(t)=g^{2}(t)$ and $b_{n}(\alpha, t)=\alpha g(t)$, where $g$ is a continuous and positive function for all $t \in I$, and $\alpha \geq 2$. Then,

$$
\begin{aligned}
& P_{2 n-1}(x, \alpha, t)=\sum_{j=0}^{n-1}(-1)^{j}\left(\begin{array}{c}
2 n-1-j \\
2(n-j)-1
\end{array}\right) g^{2 j}(t)(x-\alpha g(t))^{2(n-j)-1}, \\
& P_{2 n}(x, \alpha, t)=\sum_{j=0}^{n}(-1)^{j}(2 n-j 2(n-j)) g^{2 j}(t)(x-\alpha g(t))^{2(n-j)} .
\end{aligned}
$$

Proof. The case $n=0$ is trivial. The proof is by induction on $n \geq 1$. Again, writing $y_{\alpha, t}=x-\alpha g(t)$ and using equation (16), we obtain

$$
\begin{aligned}
P_{2 n+1}(x, \alpha, t)= & y_{\alpha, t} P_{2 n}(x, \alpha, t)-g^{2}(t) P_{2 n-1}(x, \alpha, t), \\
= & y_{\alpha, t}^{2 n+1}+\sum_{j=1}^{n}(-1)^{j}\left(\begin{array}{c}
2 n-j \\
2(n-j)
\end{array}\right) g^{2 j}(t) y_{\alpha, t}^{2(n-j)+1} \\
& +\sum_{j=1}^{n}(-1)^{j-1}\left(\begin{array}{c}
2 n-j \\
2(n-j)+1
\end{array}\right) g^{2 j}(t) y_{\alpha, t}^{2(n-j)+1}, \\
= & \left(\begin{array}{c}
2 n+1 \\
2 n+1
\end{array}\right) y_{\alpha, t}^{2 n+1}+\sum_{j=1}^{n}(-1)^{j}\left(\begin{array}{c}
2 n+1-j \\
2(n-j)+1
\end{array}\right) g^{2 j}(t) y_{\alpha, t}^{2(n-j)+1}, \\
= & \sum_{j=0}^{n}(-1)^{j}\left(\begin{array}{c}
2(n+1)-1-j \\
2(n+1-j)-1
\end{array}\right) g^{2 j}(t) y_{\alpha, t}^{2(n-j)+1},
\end{aligned}
$$

which is equation (22) when $n$ is replaced by $n+1$. Equation (23) can be obtained in a similar way.
Moreover, since $\left\{a_{n}(t)\right\}_{n \geq 0}$ and $\left\{b_{n}(\alpha, t)\right\}_{n \geq 0}$ are constant sequences, if $\alpha, t$ are fixed, and both the orthogonal and the 
second kind polynomials satisfy the same recurrence relation (with different initial conditions), it follows that $Q_{n}(x, \alpha, t)=P_{n-1}(x, \alpha, t)$ for every $n \geq 1$. Hence, it is convenient to expand equations (22) and (23) in terms of $x$.
Proposition 4. For $n \geq 0$, let $a_{n}(t)=g^{2}(t), b_{n}(\alpha, t)=\alpha g(t)$, where $g$ is a continuous and positive function for all $t \in I$, and $\alpha \geq 2$. Then,

$$
\begin{aligned}
P_{2 n}(x, \alpha, t)= & \sum_{k=0}^{n}\left(\sum_{j=1}^{n+1-k} c_{n, k+j-1}\left(\begin{array}{c}
2(k+j-1) \\
2 k
\end{array}\right)(-\alpha g(t))^{2(j-1)}\right) x^{2 k} \\
& +\sum_{k=0}^{n-1}\left(\sum_{j=1}^{n-k} c_{n, k+j}\left(\begin{array}{c}
2(k+j) \\
2 k+1
\end{array}\right)(-\alpha g(t))^{2 j-1}\right) x^{2 k+1}, \\
& P_{2 n+1}(x, \alpha, t)=\sum_{k=0}^{n}\left(\sum_{j=1}^{n+1-k} d_{n, k+j-1}\left(\begin{array}{c}
2(k+j)-1 \\
2 k
\end{array}\right)(-\alpha g(t))^{2 j-1}\right) x^{2 k} \\
& +\sum_{k=0}^{n}\left(\sum_{j=1}^{n-k} d_{n, k+j}\left(\begin{array}{c}
2(k+j)+1 \\
2 k+1
\end{array}\right)(-\alpha g(t))^{2 j}\right) x^{2 k+1},
\end{aligned}
$$

where $c_{n, k}=(-1)^{n+k}\left(\begin{array}{c}n+k \\ 2 k\end{array}\right) g^{2(n-k)}(t)$ and $d_{n, k}=(-1)^{n+k}$ $\left(\begin{array}{c}n+1+k \\ 2 k+1\end{array}\right) g^{2(n-k)}(t)$.

Proof. The result follows easily by using the binomial expansion theorem on equations (22) and (23).

As a consequence, for this particular choice of $a_{n}(t)$ and $b_{n}(\alpha, t)$, using Theorem 1 and the subsequent discussion, we can state the following result.

Proposition 5. Let $\left\{P_{n}(x, \alpha, t)\right\}_{n \geq 0}$ be the sequence of orthogonal polynomials as in Proposition 4. For $n \geq 0, t>0$, and $\alpha \geq 2$,

$f_{2 n}(x, \alpha, t)=(-1)^{n} P_{n}\left(-x^{2}, \alpha, t\right)+(-1)^{n-1} x P_{n-1}\left(-x^{2}, \alpha, t\right)$.

is a stable polynomial of degree $2 n$, where $P_{-1}(x, \alpha, t)=0$. On the other hand, fix $n$ and choose $M$ such that $P_{n-1}(0, \alpha, t) / P_{n}(0, \alpha, t)<M$ for $t>0$ and $\alpha \geq 2$. Then,

$f_{2 k+1}(x, \alpha, t)=(-1)^{k} x P_{k}\left(-x^{2}, \alpha, t\right)+(-1)^{k} G_{k}\left(-x^{2}, \alpha, t\right)$.

is a stable polynomial with degree $2 k+1$ for all $k=1, \ldots, n$. Here, $G_{k}(x, \alpha, t)=\lambda P_{k}(x, \alpha, t)+P_{k-1}(x, \alpha, t)$ with $\lambda>M$, where the coefficients of the even and odd powers of $x$ in $f_{n}(x, \alpha, t)$ are given in Proposition 4 .

We point out that since the only conditions on the function $g$ in Proposition 3 are positivity and continuity, the procedure to construct sequences of stable Hurwitz polynomials allows us to obtain different types of uncertainty. As an example, we can set $g(t)=\sin (t)$ for $t \in I=(0, \pi)$ and the obtained uncertainty structure will be nonlinear. Finally, it is also important to notice that there are many different choices for $\left\{a_{n}(t)\right\}_{n \geq 0}$ and $\left\{b_{n}(t)\right\}_{n \geq 0}$. The only condition is that they generate a chain sequence, as pointed out in the previous section. In the general case, given both sequences,
$\left\{P_{n}(x, t)\right\}_{n \geq 0}$ and $\left\{Q_{n}(x, t)\right\}_{n \geq 0}$ can be computed by using equations (7) and (8), respectively, and the robustly stable Hurwitz polynomials can be obtained by using Theorem 1 . Nevertheless, if both sequences are chosen in a simple way, as the cases considered in this section, then it is possible to get explicit expressions for the polynomials.

\section{Stable Polynomials Associated with Linear Combinations of Orthogonal Polynomials}

The particular choice of the constant sequences $\left\{a_{n}(t)\right\}_{n \geq 0}$ and $\left\{b_{n}(\alpha, t)\right\}_{n \geq 0}$ in the previous section led to the situation where $Q_{n}=P_{n-1}$, and thus in the construction of robustly stable polynomials given in Proposition 5 a linear combination of $P_{n}$ and $P_{n-1}$ appears in the polynomial $G_{n}$ used for the odd degree polynomials. In this section, we further explore the idea of using linear combinations of orthogonal polynomials to construct robustly stable sequences of Hurwitz polynomials. Recall that if $\left\{P_{n}\right\}_{n \geq 0}$ is a sequence of orthogonal polynomials, then the roots of $P_{n}$ and $P_{n-1}$ are real, simple, and interlaced. The following result establishes an interlacing property for linear combinations of orthogonal polynomials.

Theorem 2. (see [31]). For $n \geq 1$ and $\lambda_{1}, \lambda_{2} \in \mathbb{R}$ and both not zero, $\lambda_{1} P_{n}(x)+\lambda_{2} P_{n-1}(x)$ has real and simple roots. Moreover, if $\mu_{1}, \mu_{2} \in \mathbb{R}$, then $\lambda_{1} P_{n}(x)+\lambda_{2} P_{n-1}(x)$ and $\mu_{1} P_{n}(x)+\mu_{2} P_{n-1}(x)$ have no common roots and their roots satisfy an interlacing property, unless one combination is a scalar multiple of the other.

Set

$$
\begin{aligned}
& F_{n}\left(x ; \lambda_{1}, \lambda_{2}\right)=\lambda_{1} P_{n}(x)+\lambda_{2} P_{n-1}(x), \\
& G_{n}\left(x ; \mu_{1}, \mu_{2}\right)=\mu_{1} P_{n}(x)+\mu_{2} P_{n-1}(x) .
\end{aligned}
$$

$\lambda_{1}, \lambda_{2}, \mu_{1}, \mu_{2} \in \mathbb{R}$ such that $\lambda_{1} \mu_{2}-\mu_{1} \lambda_{2} \neq 0$. Then, according to the previous theorem, the roots of $F_{n}\left(x ; \lambda_{1}, \lambda_{2}\right)$ and $G_{n}\left(x ; \mu_{1}, \mu_{2}\right)$ are real, simple, and interlaced. As a 
consequence, we can use the Hermite-Biehler theorem to construct Hurwitz polynomials as in Theorem 1. Namely,

$$
f_{2 n+1}(x)=(-1)^{n} x F_{n}\left(-x^{2} ; \lambda_{1}, \lambda_{2}\right)+(-1)^{n} G_{n}\left(-x^{2} ; \mu_{1}, \mu_{2}\right)
$$

will be a Hurwitz polynomial of degree $2 n+1$ if the choice of the four parameters $\lambda_{1}, \lambda_{2}, \mu_{1}, \mu_{2} \in \mathbb{R}$ guarantees the interlacing of the roots of $F_{n}$ and $G_{n}$ and the largest root of $F_{n}$ is greater than the largest root of $G_{n}$. Notice that the latter condition implies we must have $\lambda_{1} \neq 0$ and $\mu_{1} \neq 0$. A similar approach can be taken to construct Hurwitz polynomials of even degree, but it will be necessary to set $\mu_{1}=0$ so that $G_{n}$ is a polynomial of degree $n-1$, and to write

$$
f_{2 n}(x)=(-1)^{n} F_{n}\left(-x^{2} ; \lambda_{1}, \lambda_{2}\right)+(-1)^{n} x G_{n}\left(-x^{2} ; 0, \mu_{2}\right),
$$

where we again have the requirement $\lambda_{1} \neq 0$ (which implies $\mu_{2} \neq 0$ so that $\left.\lambda_{1} \mu_{2}-\mu_{1} \lambda_{2} \neq 0\right)$. Observe that we can consider $\lambda_{1}, \lambda_{2}, \mu_{1}, \mu_{2} \in \mathbb{R}$ as uncertain parameters, and by deducing appropriate ranges of values for each one of them such that the interlacing property of the roots of $F_{n}$ and $G_{n}$ is satisfied, the Hermite-Biehler theorem guarantees that the constructed polynomials will be robustly stable for all values of the parameters within an specified range. In this sense, we will determine such conditions on the four parameters. Observe that the even and odd degree cases are independent and thus will be analyzed separately. We begin with the even degree case. Recall that the sign function is denoted by

$$
\operatorname{sgn}[x]= \begin{cases}1 & \text { if } x>0, \\ 0 & \text { if } x=0, \\ -1 & \text { if } x<0 .\end{cases}
$$

Proposition 6. Let $\left\{P_{n}(x)\right\}_{n \geq 0}$ be a sequence of monic polynomials orthogonal with respect to some positive measure supported in some interval $E \subset \mathbb{R}_{+}$, and let $F_{n}\left(x ; \lambda_{1}, \lambda_{2}\right)$ and $G_{n}\left(x ; 0, \mu_{2}\right)\left(\right.$ i.e. $\left.\mu_{1}=0\right)$ be defined as above. Then, for $n \geq 1$,

$$
f_{2 n}(x)=(-1)^{n} \operatorname{sgn}\left[\lambda_{1}\right] F_{n}\left(-x^{2} ; \lambda_{1}, \lambda_{2}\right)+(-1)^{n-1} \operatorname{sgn}\left[\mu_{2}\right] x G_{n}\left(-x^{2} ; 0, \mu_{2}\right) .
$$

is a Hurwitz polynomial of degree $2 n$ for every $\lambda_{1} \neq 0, \mu_{2} \neq 0$, $\operatorname{sgn}\left[G_{n}\left(x_{n, n}^{\lambda_{1}, 0} ; \mu_{1}, \mu_{2}\right)\right]=\operatorname{sgn}\left[\mu_{2}\right] \operatorname{sgn}\left[P_{n-1}\left(x_{n, n}^{\lambda_{1}, 0}\right)\right]=\operatorname{sgn}\left[\mu_{2}\right]$.

$$
\lambda_{2}<-\frac{\left|\lambda_{1}\right|}{\operatorname{sgn}\left[\lambda_{1}\right]} \frac{P_{n}(0)}{P_{n-1}(0)} .
$$

Proof. Since $\lambda_{1}, \mu_{2}$ are nonzero and $n \geq 1$, $\operatorname{sgn}\left[\lambda_{1}\right] F_{n}\left(x ; \lambda_{1}, 0\right)=\left|\lambda_{1}\right| P_{n}(x)$ and $P_{n-1}(x)$ have positive, simple, and interlacing zeros, and thus their coefficients have alternating signs. Hence, both $(-1)^{n-1}\left|\mu_{2}\right| P_{n-1}(-x)$ and

$$
(-1)^{n}\left|\lambda_{1}\right| P_{n}(-x)+(-1)^{n} \operatorname{sgn}\left[\lambda_{1}\right] \lambda_{2} P_{n-1}(-x)
$$

have negative, simple, and interlacing zeros and positive coefficients, provided that $\lambda_{2}$ satisfies $\operatorname{sgn}\left[\lambda_{1}\right] \lambda_{2}<-\left|\lambda_{1}\right| P_{n}(0) / P_{n-1}(0)$. The result follows from the Hermite-Biehler theorem.

Now we consider the odd degree case. Recall that in this situation we must have $\lambda_{1} \neq 0$ and $\mu_{1} \neq 0$. Given $n \geq 1$, let us denote by $x_{n, 1}^{\lambda_{1}, \lambda_{2}}<\ldots<x_{n, n}^{\lambda_{1}, \lambda_{2}}$ and $y_{n, 1}^{\mu_{1}, \mu_{2}}<\ldots<y_{n, n}^{\mu_{1}, \mu_{2}}$ the roots of $F_{n}\left(x ; \lambda_{1}, \lambda_{2}\right)$ and $G_{n}\left(x ; \mu_{1}, \mu_{2}\right)$, respectively, in increasing order.

Assume first $\lambda_{2}=0$. As stated in Section 1, if the orthogonality measure is supported in $E \subset \mathbb{R}_{+}$, then the roots of $F_{n}\left(x ; \lambda_{1}, 0\right)=\lambda_{1} P_{n}(x)$ belong to the interior of the convex hull of $E$ and interlace with the roots of $G_{n}\left(x ; \mu_{1}, \mu_{2}\right)$ when $\lambda_{1} \mu_{2} \neq 0$, from Theorem 2 . Hence, our next goal is to determine when the largest root of $F_{n}\left(x ; \lambda_{1}, 0\right)$ is greater than the largest root of $G_{n}\left(x ; \mu_{1}, \mu_{2}\right)$ in terms of the parameters $\lambda_{1}, \mu_{1}, \mu_{2}, \in \mathbb{R}$. Furthermore, in this situation the roots of $F_{n}\left(x ; \lambda_{1}, 0\right)$ also interlace with the roots of $P_{n-1}$, and therefore $\operatorname{sgn}\left[P_{n-1}\left(x_{n, n}^{\lambda_{1}, 0}\right)=1\right]$. Thus, for all $\mu_{1}, \mu_{2} \in \mathbb{R}$,

As a consequence, for $\lambda_{1} \mu_{2} \neq 0$, we get the following.

(1) If $\lambda_{2}=0, \mu_{1}>0$ then

$$
\operatorname{sgn}\left[\mu_{2}\right] y_{n, k}^{\mu_{1}, \mu_{2}}<\operatorname{sgn}\left[\mu_{2}\right] x_{n, k}^{\lambda_{1}, 0} .
$$

(2) If $\lambda_{2}=0, \mu_{1}<0$ then

$$
\operatorname{sgn}\left[\mu_{2}\right] x_{n, k}^{\lambda_{1}, 0}<\operatorname{sgn}\left[\mu_{2}\right] y_{n, k}^{\mu_{1}, \mu_{2}} .
$$

We now turn to the case $\lambda_{2} \neq 0$. Notice that the continuity of the determinant $\lambda_{1} \mu_{2}-\mu_{1} \lambda_{2} \neq 0$ together with Theorem 2 implies that equations (36) and (37) still hold when $\lambda_{2} \neq 0$, as long as the determinant is nonzero. Thus, we consider the following four cases:

(3) If $\lambda_{2}>0$ and $\mu_{1}>0$, then

$$
\operatorname{sgn}\left[\frac{\mu_{1} \lambda_{2}}{\lambda_{1}}-\mu_{2}\right] x_{n, k}^{\lambda_{1}, \lambda_{2}}<\operatorname{sgn}\left[\frac{\mu_{1} \lambda_{2}}{\lambda_{1}}-\mu_{2}\right] y_{n, k}^{\mu_{1}, \mu_{2}} .
$$

(4) If $\lambda_{2}>0$ and $\mu_{1}<0$, then

$$
\operatorname{sgn}\left[\frac{\mu_{1} \lambda_{2}}{\lambda_{1}}-\mu_{2}\right] y_{n, k}^{\mu_{1}, \mu_{2}}<\operatorname{sgn}\left[\frac{\mu_{1} \lambda_{2}}{\lambda_{1}}-\mu_{2}\right] x_{n, k}^{\lambda_{1}, \lambda_{2}} .
$$

(5) If $\lambda_{2}<0$ and $\mu_{1}>0$, then

$$
\operatorname{sgn}\left[\frac{\mu_{1} \lambda_{2}}{\lambda_{1}}-\mu_{2}\right] x_{n, k}^{\lambda_{1}, \lambda_{2}}<\operatorname{sgn}\left[\frac{\mu_{1} \lambda_{2}}{\lambda_{1}}-\mu_{2}\right] y_{n, k}^{\mu_{1}, \mu_{2}} .
$$

(6) If $\lambda_{2}<0$ and $\mu_{1}<0$, then 


$$
\operatorname{sgn}\left[\frac{\mu_{1} \lambda_{2}}{\lambda_{1}}-\mu_{2}\right] y_{n, k}^{\mu_{1}, \mu_{2}}<\operatorname{sgn}\left[\frac{\mu_{1} \lambda_{2}}{\lambda_{1}}-\mu_{2}\right] x_{n, k}^{\lambda_{1}, \lambda_{2}} .
$$

Clearly, equations (36), (38), and (40) (resp. equations (37), (39), and (41)) imply that the inequality for the case $\mu_{1}>0$ (resp. $\mu_{1}<0$ ) is independent of value of $\lambda_{2}$. As a consequence, we can now proceed with the construction of sequences of Hurwitz polynomials, by using the Hermite-Biehler theorem and the interlacing property of the zeros of $F_{n}\left(x ; \lambda_{1}, \lambda_{2}\right)$ and $G_{n}\left(x ; \mu_{1}, \mu_{2}\right)$. Recall that, for the odd degree case, the construction of a Hurwitz polynomial depends of the relative position of the zeros of $F_{n}\left(x ; \lambda_{1}, \lambda_{2}\right)$ and $G_{n}\left(x ; \mu_{1}, \mu_{2}\right)$ and therefore of the values of parameters $\lambda_{1}, \lambda_{2}, \mu_{2}, \mu_{1}$.

Proposition 7. Let $\left\{P_{n}(x)\right\}_{n \geq 0}$ be a sequence of monic polynomials orthogonal with respect to some positive measure supported in some interval $E \subset \mathbb{R}_{+}$, and let $F_{n}\left(x ; \lambda_{1}, \lambda_{2}\right)$ and $G_{n}\left(x ; \mu_{1}, \mu_{2}\right)$ be defined as above, with $\lambda_{1} \neq 0$ and $\mu_{1} \neq 0$. Then, for $n \geq 1$, (i) if $\mu_{1}>0$ and $\lambda_{2} \in \mathbb{R}$ such that $\operatorname{sgn}\left[\lambda_{1}\right] \lambda_{2}<-\left|\lambda_{1}\right| P_{n}(0) / P_{n-1}(0)$, then

$(-1)^{n} \operatorname{sgn}\left[\lambda_{1}\right] x F_{n}\left(-x^{2} ; \lambda_{1}, \lambda_{2}\right)+(-1)^{n} G_{n}\left(-x^{2} ; \mu_{1}, \mu_{2}\right)$.

is a Hurwitz polynomial of degree $2 n+1$ if and only if

$$
\frac{\mu_{1} \lambda_{2}}{\lambda_{1}}<\mu_{2}<-\mu_{1} \frac{P_{n}(0)}{P_{n-1}(0)} \text {. }
$$

Also,

$$
(-1)^{n} x G_{n}\left(-x^{2} ; \mu_{1}, \mu_{2}\right)+(-1)^{n} \operatorname{sgn}\left[\lambda_{1}\right] F_{n}\left(-x^{2} ; \lambda_{1}, \lambda_{2}\right) .
$$

is a Hurwitz polynomial of degree $2 n+1$ if and only if

$$
\mu_{2}<\min \left\{\frac{\mu_{1} \lambda_{2}}{\lambda_{1}},-\mu_{1} \frac{P_{n}(0)}{P_{n-1}(0)}\right\} \text {. }
$$

(ii) if $\mu_{1}<0$ and $\lambda_{2} \in \mathbb{R}$ such that $\operatorname{sgn}\left[\lambda_{1}\right] \lambda_{2}<-\left|\lambda_{1}\right| P_{n}(0) / P_{n-1}(0)$, then

$$
(-1)^{n} \operatorname{sgn}\left[\lambda_{1}\right] x F_{n}\left(-x^{2} ; \lambda_{1}, \lambda_{2}\right)+(-1)^{n} \operatorname{sgn}\left[\mu_{1}\right] G_{n}\left(-x^{2} ; \mu_{1}, \mu_{2}\right) .
$$

is a Hurwitz polynomial of degree $2 n+1$ if and only if

Also,

$$
-\mu_{1} \frac{P_{n}(0)}{P_{n-1}(0)}<\mu_{2}<\frac{\mu_{1} \lambda_{2}}{\lambda_{1}} .
$$

$$
(-1)^{n} \operatorname{sgn}\left[\mu_{1}\right] x G_{n}\left(-x^{2} ; \mu_{1}, \mu_{2}\right)+(-1)^{n} \operatorname{sgn}\left[\lambda_{1}\right] F_{n}\left(-x^{2} ; \lambda_{1}, \lambda_{2}\right) .
$$

is a Hurwitz polynomial of degree $2 n+1$ if and only if

$$
\mu_{2}>\max \left\{\frac{\mu_{1} \lambda_{2}}{\lambda_{1}},-\mu_{1} \frac{P_{n}(0)}{P_{n-1}(0)}\right\} .
$$

Proof. Assume $\mu_{1}>0$. The proof is based on the observation that both $(-1)^{n} \operatorname{sgn}\left[\lambda_{1}\right] F_{n}\left(-x ; \lambda_{1}, \lambda_{2}\right)$ and $(-1)^{n} G_{n}$ $\left(-x ; \mu_{1}, \mu_{2}\right)$ have negative, simple, and interlacing zeros, and thus their coefficients are positive if and only if $\operatorname{sgn}\left[\lambda_{1}\right] \lambda_{2}<-\left|\lambda_{1}\right| P_{n}(0) / P_{n-1}(0)$ and $\mu_{1} \lambda_{2} / \lambda_{1} \neq \mu_{2}<-$ $\mu_{1} P_{n}(0) / P_{n-1}(0)$, respectively. Indeed, if it were true that $\operatorname{sgn}\left[\lambda_{1}\right] \lambda_{2}<-\left|\lambda_{1}\right| P_{n}(0) / P_{n-1}(0)$ and $\mu_{2}=-\mu_{1} P_{n}(0) / P_{n-1}$ (0), we would have $F_{n}\left(0 ; \lambda_{1}, \lambda_{2}\right)=0$ and $G_{n}\left(0 ; \mu_{1}, \mu_{2}\right)=0$. Moreover, the fact that both $F_{n}\left(x ; \lambda_{1}, \lambda_{2}\right)$ and $G_{n}\left(x ; \mu_{1}, \mu_{2}\right)$ have the same roots follows from Theorem 2 if $\mu_{2}=\mu_{1} \lambda_{2} / \lambda_{1}$. Therefore, since $E \subset \mathbb{R}_{+}$, we conclude that $0<y_{n, k}^{\mu_{1}, \mu_{2}}<x_{n, k}^{\lambda_{1}, \lambda_{2}}$ (resp. $0<x_{n, k}^{\lambda_{1}, \lambda_{2}}<y_{n, k}^{\mu_{1}, \mu_{2}}$ ) for all $k=1, \ldots, n$ if $\mu_{1} \lambda_{2} / \lambda_{1}<\mu_{2}$ (resp. $\mu_{2}<\mu_{1} \lambda_{2} / \lambda_{1}$ ). Hence $i$ ) follows from the Hermite-Biehler theorem. A similar argument can be applied to the case when $\mu_{1}<0$.

\section{Numerical Examples}

In this section we provide some numerical examples, computed using Mathematica ${ }^{\circledR}$, to illustrate the behavior of the roots of stable polynomials generated by the three-term relation equation (13) and stable polynomials associated with linear combinations of orthogonal polynomials. More specifically, we will plot the location of the roots of some polynomials given by Propositions 5 and 7 for some values of the parameters.

First, consider the case when $g(t)=t^{1 / 2}, t \in(0, \infty)$, and $\alpha \geq 2$ in Proposition 5. Then, the first even degree polynomials are 


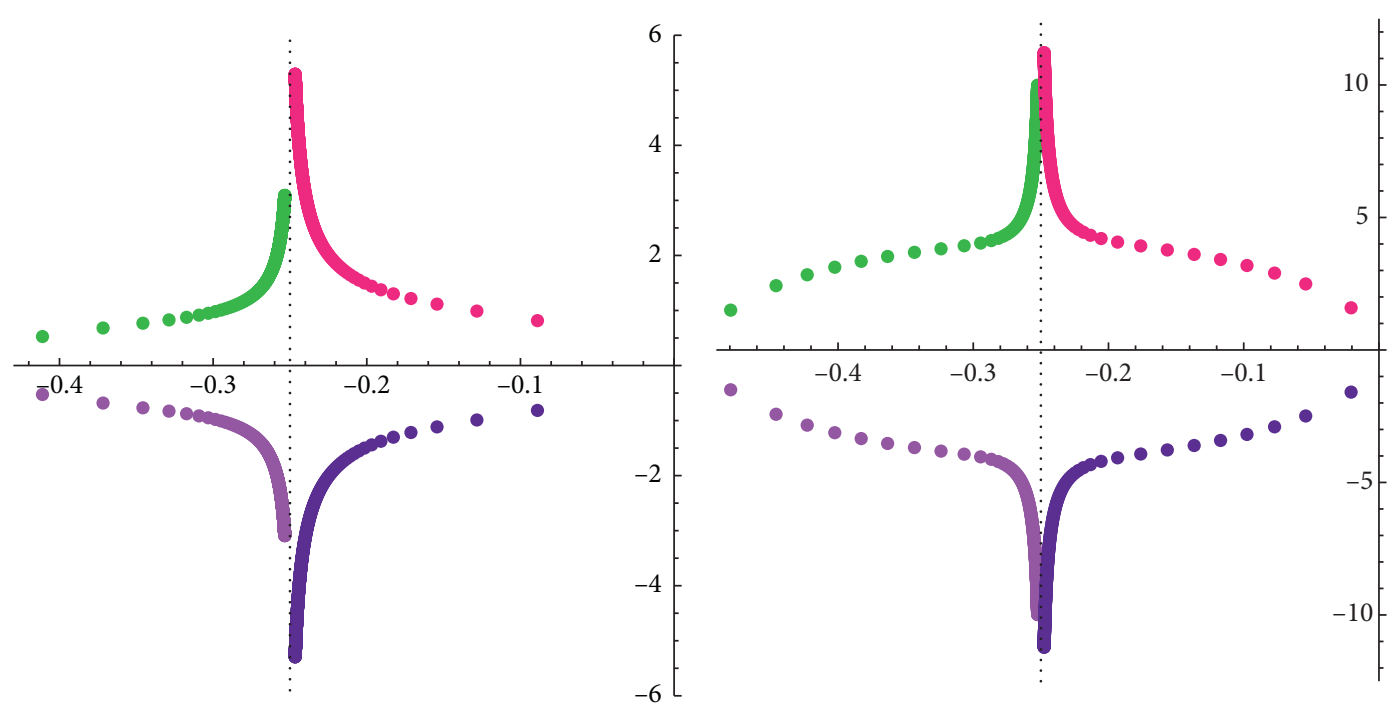

(a)

(b)

FIgURE 1: (a) The location of the roots of $f_{4}(x, 2, t)$ for several values of $t$. (b) The location of the roots of $f_{4}(x, 8, t)$ for several values of $t$.

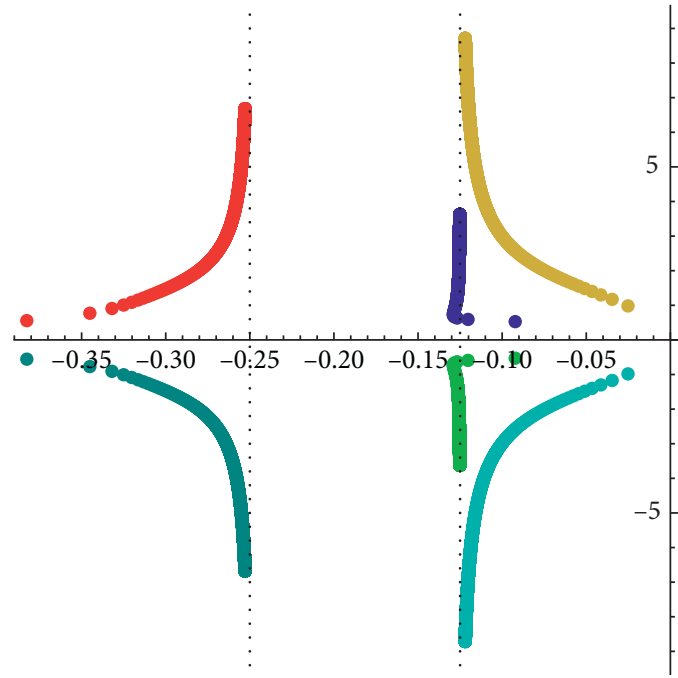

(a)

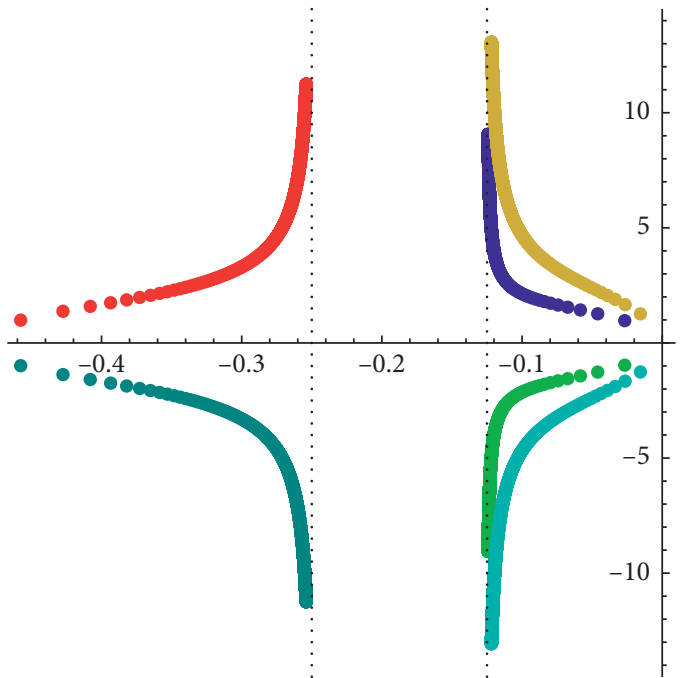

(b)

Figure 2: (a) Location of the roots of $f_{6}(x, 2, t)$ for several values of $t$. (b) Location of the roots of $f_{6}(x, 4, t)$ for several values of $t$.

$$
\begin{aligned}
& f_{2}(x, \alpha, t)=x^{2}+x+\alpha t^{1 / 2} \\
& f_{4}(x, \alpha, t)=x^{4}+x^{3}+2 \alpha t^{1 / 2} x^{2}+\alpha t^{1 / 2} x+\left(\alpha^{2}-1\right) t^{1 / 2} \\
& f_{6}(x, \alpha, t)=x^{6}+x^{5}+3 \alpha t^{1 / 2} x^{4}+2 \alpha t^{1 / 2} x^{3}+\left(3 \alpha^{2}-2\right) t x^{2}+\left(\alpha^{2}-1\right) t x+\alpha\left(\alpha^{2}-2\right) t^{3 / 2} .
\end{aligned}
$$

And we obtain polynomic uncertainty in the parameter $q_{1}=\alpha$ and nonlinear uncertainty in the parameter $q_{2}=t$.

Example 1. For our first example, Figure 1 illustrates the motion of the roots of $f_{4}(x, \alpha, t)$ when $\alpha=2$ and $t$ varies from 0.1 to 90 with a step size of 0.1 (panel (a)) and when $\alpha=8$ and $t$ varies from 0.1 to 200 with a step size of 0.5 (panel (b)). For both cases, notice that the real part of all the complex roots tends approximately to $-1 / 4$ when $t \longrightarrow 90$ and $t \longrightarrow 200$, respectively.

Example 2. Proceeding in a similar way, Figure 2 shows the location of the roots of $f_{6}(x, \alpha, t)$ when $\alpha=2$ and $t$ varies from 0.1 to 500 with a step size of 0.1 (panel (a)) and when $\alpha=4$ and $t$ varies from 0.1 to 1000 with a step size of 0.2 (panel (b)). Analogously, the real part of each pair of 


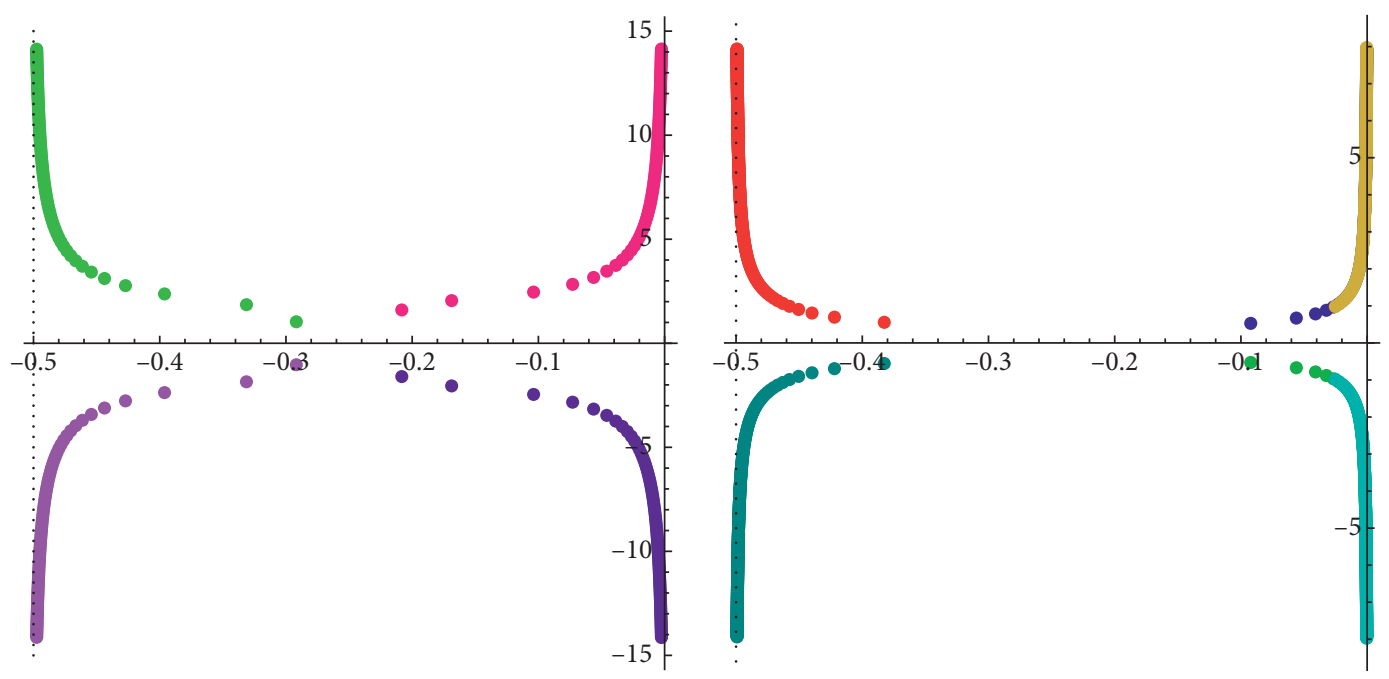

(a)

(b)

Figure 3: (a) The location of the roots of $f_{4}(x, \alpha, 1)$ for several values of $\alpha$. (b) The location of the roots of $f_{6}(x, \alpha, 0.1)$ for several values of $\alpha$.

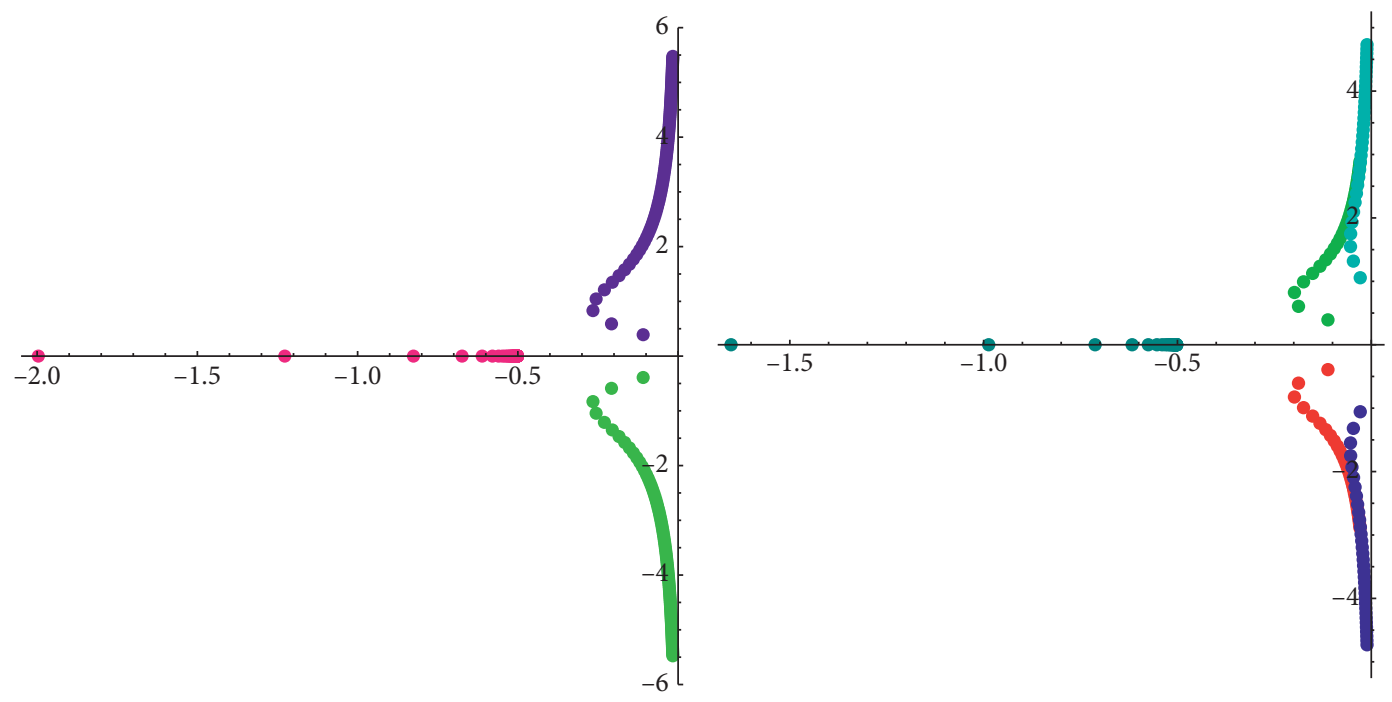

(a)

(b)

Figure 4: Motion of the complex roots of two stable polynomials of degrees three (a) and five (b) constructed via Proposition 5 for several values of $t$.

conjugate complex roots tends to two distinct negative numbers when $t \longrightarrow 500$ and $t \longrightarrow 1000$, which approximately are $-1 / 4$ and $-1 / 8$, respectively.

Example 3. In Figure 3 we present a comparison between the evolution of the roots of $f_{4}(x, \alpha, t)$ and $f_{6}(x, \alpha, t)$ with respect to $\alpha$ (with $t$ fixed). The panel (a) corresponds to the case when $t=1$ and $\alpha$ varies from 2 to 200 with a step size of 2 , while the graphic on the right corresponds to the case when $t=0.1$ and $\alpha$ varies from 2 to 200 with a step size of 0.5 . In both cases, notice that the real part of all the complex roots approximately tends to $-1 / 2$ or zero when $\alpha \longrightarrow 200$.

On the other hand, to construct stable polynomials of degrees 3 and 5 , we must compute $-P_{n-1}(0, \alpha, t) / P_{n}(0, \alpha, t)$ when $n=2$. In Proposition 5, take $g(t)=t, t \in(0, \infty)$, and $\alpha \geq 2$. Since $P_{1}(x, \alpha, t)=x-\alpha t \quad$ and $P_{2}(x, \alpha, t)=$ $x^{2}-2 \alpha t x+\left(\alpha^{2}-1\right) t^{2}$, the corresponding polynomials are

$$
\begin{aligned}
f_{1}(x, \alpha, t)= & x+\lambda \\
f_{3}(x, \alpha, t)= & x^{3}+\lambda x^{2}+\alpha t x+\lambda \alpha t-1, \\
f_{5}(x, \alpha, t)= & x^{5}+\lambda x^{4}+2 \alpha t x^{3}+(2 \lambda \alpha t-1) x^{2} \\
& +\left(\alpha^{2}-1\right) t^{2} x+\left(\alpha^{2}-1\right) \lambda t^{2}-\alpha t,
\end{aligned}
$$

where $\lambda>\alpha\left(\alpha^{2}-1\right)^{-1} t^{-1}$.

Example 4. We choose $\lambda=\lambda(\alpha, t)=0.5+\alpha\left(\alpha^{2}-1\right)^{-1} t^{-1}$; then panel (a) in Figure 4 illustrates the location of the roots of 


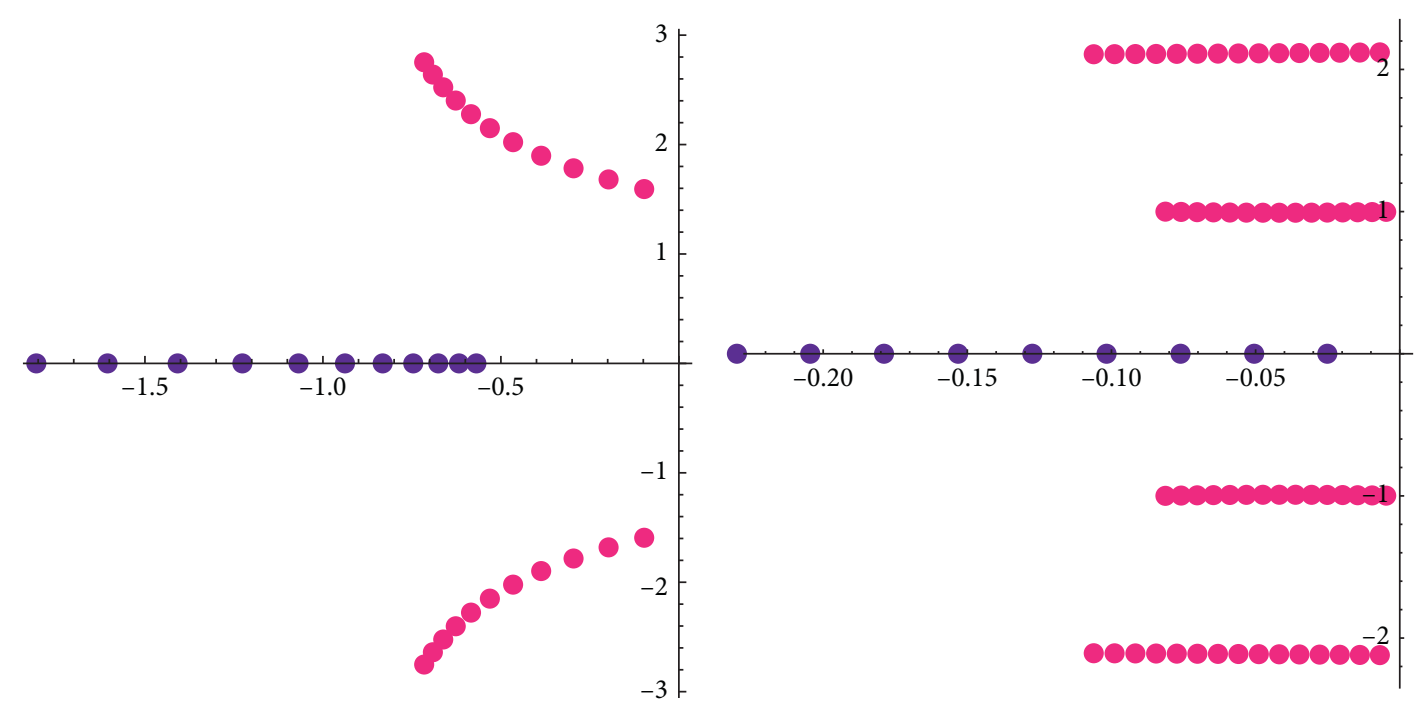

(a)

(b)

Figure 5: Motion of the complex roots of a stable polynomial of degrees three (a) and five (b) constructed via Proposition 7 for several values of $\mu_{2}$.

$f_{3}(x, 6, t)$ when $t$ varies from 0.1 to 5 with a step size of 0.05 and panel (b) in Figure 4 shows the motion of the roots of $f_{5}(x, 2.2, t)$ when $t$ varies from 0.4 to 7 with a step size of 0.2 . For both cases, the single real root tends to $\lim _{t \rightarrow \infty}-\lambda(\alpha, t)=$ -0.5 and the real part of all the complex roots tends approximately to 0 when $t \longrightarrow 5$ and $t \longrightarrow 7$, respectively.

Finally, we now show an example of robustly stable polynomials constructed by using linear combinations of Laguerre polynomials, which are orthogonal with respect to the measure $x^{\alpha} e^{-x}$ with support in $\mathbb{R}_{+}$and defined for $\alpha>-1$. In particular, the first Laguerre monic polynomials are

$$
\begin{aligned}
L_{1}^{\alpha}(x)= & x-(\alpha+1), \\
L_{2}^{\alpha}(x)= & x^{2}-2(\alpha+2) x+\alpha^{2}+3 \alpha+2, \\
L_{3}^{\alpha}(x)= & x^{3}-3(\alpha+3) x^{2}+3\left(\alpha^{2}+5 \alpha+6\right) x-\alpha^{3} \\
& +6 \alpha^{2}+11 \alpha+6 .
\end{aligned}
$$

Example 5. Taking into account these polynomials, Figure 5 illustrates the motion of the roots of a stable polynomial of degree 3 constructed with $\alpha=2, \lambda_{1}=1, \lambda_{2}=0.7$, and $\mu_{1}=$ 0.5 (panel (a)). According to Proposition 7, $\mu_{2}$ can vary from -2.95 to 0.05 , and the roots are plotted using a step size of 0.3 . Similarly, panel (b) in Figure 5 corresponds to the case when $\alpha=1 / 2, \lambda_{1}=1, \lambda_{2}=-0.5, \mu_{1}=-0.4$, and $\mu_{2}$ varies from -0.925 to 0.125 with a step size of 0.15 .

\section{Discussion and Further Comments}

We have used algebraic properties of orthogonal polynomials to construct families of Hurwitz polynomials that are robustly stable in terms of a set of parameters. First, we use Favard's theorem to introduce $t$ and $\alpha$ as parameters in the coefficients of the recurrence relation, which are now continuous functions of those parameters. Notice that there is a great deal of generality on the choice of $a_{n}(t)$ and $b_{n}(\alpha, t)$. Indeed, the only requirement is that they generate a chain sequence, as mentioned in Section 2. Of course, one can introduce an arbitrary set of parameters, say $a_{n}\left(t_{1}, \ldots, t_{k}\right), b_{n}\left(\widetilde{t}_{1}, \ldots, \widetilde{t}_{l}\right)$, as long as the corresponding sequences generate a chain sequence for all values of all parameters within a specified range.

On the other hand, we have also shown that the Hermite-Biehler theorem can be used to construct Hurwitz polynomials from linear combinations of two consecutive degree orthogonal polynomials, whose roots satisfy an interlacing property. Here, four real parameters are considered and Hurwitz polynomials of even and odd degree can be constructed if the parameters satisfy some inequalities.

As a consequence of the results presented here, together with those of the recent contribution [29], it is possible to use orthogonal polynomials to construct sequences of robustly stable Hurwitz polynomials in three different ways: by introducing an uncertain parameter on the orthogonality measure, by introducing uncertain parameters on the threeterm recurrence relation, and by using linear combinations of orthogonal polynomials. The results of this work can be extended in the following directions:

(1) It is possible to use Hurwitz polynomials constructed by using orthogonal polynomials in pole-placement design: to develop a controller that yields a closedloop system with a prescribed characteristic polynomial [32, 33]. In this situation, an interesting open problem is to analyze the capabilities of the resulting system, such as closed-loop frequency response, disturbance rejection, and sensitivity [34]. It is worth noticing that properties of orthogonal polynomials have been used recently to propose a stabilization method for interval plants with uncertain time-delay by using the set value concept [30].

(2) Although the proposed Hurwitz polynomials are robustly stable for the prescribed values of the 
parameters, an interesting open problem is to deduce the equations of motion of the roots in terms of the parameters (i.e., their behavior in terms of the involved parameters or, equivalently, the algebraic expressions for the curves they describe on the complex plane), as well as to determine the regions of the complex plane where the roots will be located for every value of the parameters. This information would be useful in the design of controllers in poleplacement design previously mentioned.

\section{Data Availability}

No data were used to support this study.

\section{Conflicts of Interest}

The authors declare that they have no conflicts of interest.

\section{Acknowledgments}

The work of the third author was supported by Universidad de Monterrey. The work of the fourth author was supported by México's CONACYT under Grant 287523. The work of third and fourth authors was partially funded by Dirección General de Investigación e Innovación, Consejería de Educación e Investigación of the Comunidad de Madrid (Spain), and Universidad de Alcalá under grant CM/JIN/ 2021-014, Línea de actuación estímulo a la investigación, Proyectos de $\mathrm{I}+\mathrm{D}$ para Jóvenes Investigadores de la Universidad de Alcalá 2021. The work of the fifth author was partially supported by Universidad Autónoma de Tamaulipas, under Grant PEI-PROINNOVA-2018-250117.

\section{References}

[1] J. C. Maxwell, "On governors," Proceedings of the Royal Socity, vol. 16, pp. 270-283, 1868.

[2] F. R. Gantmacher, The theory of matrices, Chelsea Publishing Co., vol. 1, p. 2, New York, USA, 1959.

[3] P. Lancaster and M. Tismenetsky, "The Theory of Matrices," Computer Science and Applied Mathematics, Academic Press, Orlando, Florida, Second edition, 1985.

[4] O. Holtz, "Hermite-Biehler, Routh-Hurwitz, and total positivity," Linear Algebra and Its Applications, vol. 372, pp. 105-110, 2003.

[5] S. P. Bhattacharyya, H. Chapellat, and L. H. Keel, Robust Control: The Parametric Approach, Prentice-Hall, Upper Saddle River, NJ, USA, 1995.

[6] C. Hermite, "Sur le nombre des racines d'une équation algébrique comprise entre des limites données," Journal für die Reine und Angewandte Mathematik, vol. 52, pp. 39-51, 1856.

[7] V. L. Kharitonov, "Asymptotic stability of an equilibrium position of a family of systems of linear differential equations," Dif. Urav.vol. 14, pp. 1483-1485, 1979.

[8] A. C. Bartlett, C. V. Hollot, and H. Lin, "Root locations of an entire polytope of polynomials: it suffices to check the edges," in Proceedings of the 1987 American Control Conference, vol. 1, pp. 61-71, Minneapolis, MN, USA, June, 1987.
[9] B. R. Barmish, New Tools for Robustness of Linear Systems, Macmillan Publishing Co., New York, NY, USA, 1994.

[10] A. E. Choque Rivero, "The Kharitonov theorem and robust stabilization via orthogonal polynomials," Visn. Khark. Univ., Ser. Mat. Prykl. Mat. Mekh.vol. 86, pp. 49-68, 2017.

[11] C. Brezinski, "Padé-type approximation and general orthogonal polynomials," International Series of Numerical Mathematics, SO. Birkhäuser Verlag, Basel-Boston, Mass. USA, 1980.

[12] T. J. Stieltjes, "Quelques recherches sur la théorie des quadratures dites mécaniques," Annales Scientifiques de l'Ecole Normale Superieure, vol. 1, no. 3, pp. 409-426, 1884.

[13] A. A. Markov, "Sur les racines de certaines équations," Mathematische Annalen, vol. 27, pp. 177-182, 1886.

[14] P. L. Chebyshev, "Sur les valeurs limites des intégrales," Jurnal Matematika, vol. 19, no. 2, pp. 157-160, 1874.

[15] W. Gautschi, "Orthogonal polynomials: applications and computation," Acta Numerica, vol. 5, pp. 45-119, 1996.

[16] C. Chuan-Tsung, A. Mironov, A. Morozov, and A. Sleptsov, "Orthogonal polynomials in mathematical physics," Reviews in Mathematical Physics, vol. 30, no. 6, pp. 119-182, 2018.

[17] R. Mukundan, S. H. Ong, and P. A. Lee, "Image analysis by Tchebichef moments," IEEE Transactions on Image Processing, vol. 10, no. 9, pp. 1357-1364, 2001.

[18] D. Rivero-Castillo, H. Pijeira, and P. Assunçao, "Edge detection based on Krawtchouk polynomials," Journal of Computational and Applied Mathematics, vol. 284, pp. 244-250, 2015.

[19] W. Schoutens, "An application in stochastics of the Laguerretype polynomials," Journal of Computational and Applied Mathematics, vol. 133, no. 1-2, pp. 593-600, 2001.

[20] S. Lawi, "Hermite and Laguerre polynomials and matrixvalued stochastic processes," Electronic Communications in Probability, vol. 13, pp. 67-84, 2008.

[21] H. Rauhut and R. Ward, "Sparse legendre expansions via $\ell 1$ minimization," Journal of Approximation Theory, vol. 164, no. 5, pp. 517-533, 2012.

[22] A. Sandryhaila, J. Kovacevic, and M. Puschel, "Algebraic signal processing theory: 1-D nearest neighbor models," IEEE Transactions on Signal Processing, vol. 60, no. 5, pp. 22472259, 2012.

[23] K. B. Datta and B. M. Mohan, "Orthogonal functions in systems and control," Advanced Series in Electrical and Computer Engineering, World Scientific, vol. 9, Singapore, , 1995.

[24] T. S. Chihara, An Introduction to Orthogonal Polynomials. Mathematics and its Applications Series, Gordon \& Breach, New York, USA, 1978.

[25] G. Szegö, "Orthogonal Polynomials," Amer. Math. Soc. Colloq. Publ. Series, Vol. 23, Amer. Math. Soc., Providence, Rhode Island, 4thed edition, 1975.

[26] N. Martínez, L. E. Garza, and B. Aguirre-Hernández, "On sequences of Hurwitz polynomials related to orthogonal polynomials," Linear and Multilinear Algebra, vol. 67, no. 11, pp. 2191-2208, 2019.

[27] Y. V. Genin, "Euclid algorithm, orthogonal polynomials, and generalized Routh-Hurwitz algorithm," Linear Algebra and Its Applications, vol. 246, pp. 131-158, 1996.

[28] A. E. Choque Rivero, "On matrix Hurwitz type polynomials and their interrelations to Stieltjes positive definite sequences and orthogonal matrix polynomials," Linear Algebra and Its Applications, vol. 476, pp. 56-84, 2015.

[29] A. Arceo, L. E. Garza, and G. Romero, "Robust stability of Hurwitz polynomials associated with modified classical weights," Mathematics, vol. 7, no. 9, p. 818, 2019. 
[30] P. Zamora, A. Arceo, N. Martínez, G. Romero, and L. E. Garza, "Robust stabilization of interval plants with uncertain time-delay using the value set concept," Mathematics, vol. 9, no. 4, p. 429, 2021.

[31] A. F. Beardon and K. A. Driver, "The zeros of linear combinations of orthogonal polynomials," Journal of Approximation Theory, vol. 137, no. 2, pp. 179-186, 2005.

[32] C. T. Chen, "Linear systems: theory and design," in Electrical and Computer Engineering, Oxford University Press, New York, NY, USA, 3th ed. edition, 1999.

[33] K. J. Åström and B. Wittenmark, "Computer-controller systems: theory and design," in Prentice Hall Information and System Sciences, Prentice-Hall, Upper Saddle River, NJ, USA, 3th ed. edition, 1997.

[34] C. L. Phillips and R. D. Harbor, Feedback Control Systems, Prentice-Hall, Englewood Cliffs, NJ, USA, 3th ed. edition, 1996. 